This item was submitted to Loughborough's Research Repository by the author.

Items in Figshare are protected by copyright, with all rights reserved, unless otherwise indicated.

\title{
Phi-array: a novel method for fitness visualization and decision making in evolutionary design optimization
}

PLEASE CITE THE PUBLISHED VERSION

http://dx.doi.org/10.1016/j.aei.2011.07.005

PUBLISHER

(C) Elsevier

VERSION

AM (Accepted Manuscript)

LICENCE

CC BY-NC-ND 4.0

\section{REPOSITORY RECORD}

Mourshed, Monjur, Shariful H. Shikder, and Andrew D.F. Price. 2019. "Phi-array: A Novel Method for Fitness Visualization and Decision Making in Evolutionary Design Optimization". figshare. https://hdl.handle.net/2134/9713. 
This item was submitted to Loughborough's Institutional Repository (https://dspace.lboro.ac.uk/) by the author and is made available under the following Creative Commons Licence conditions.

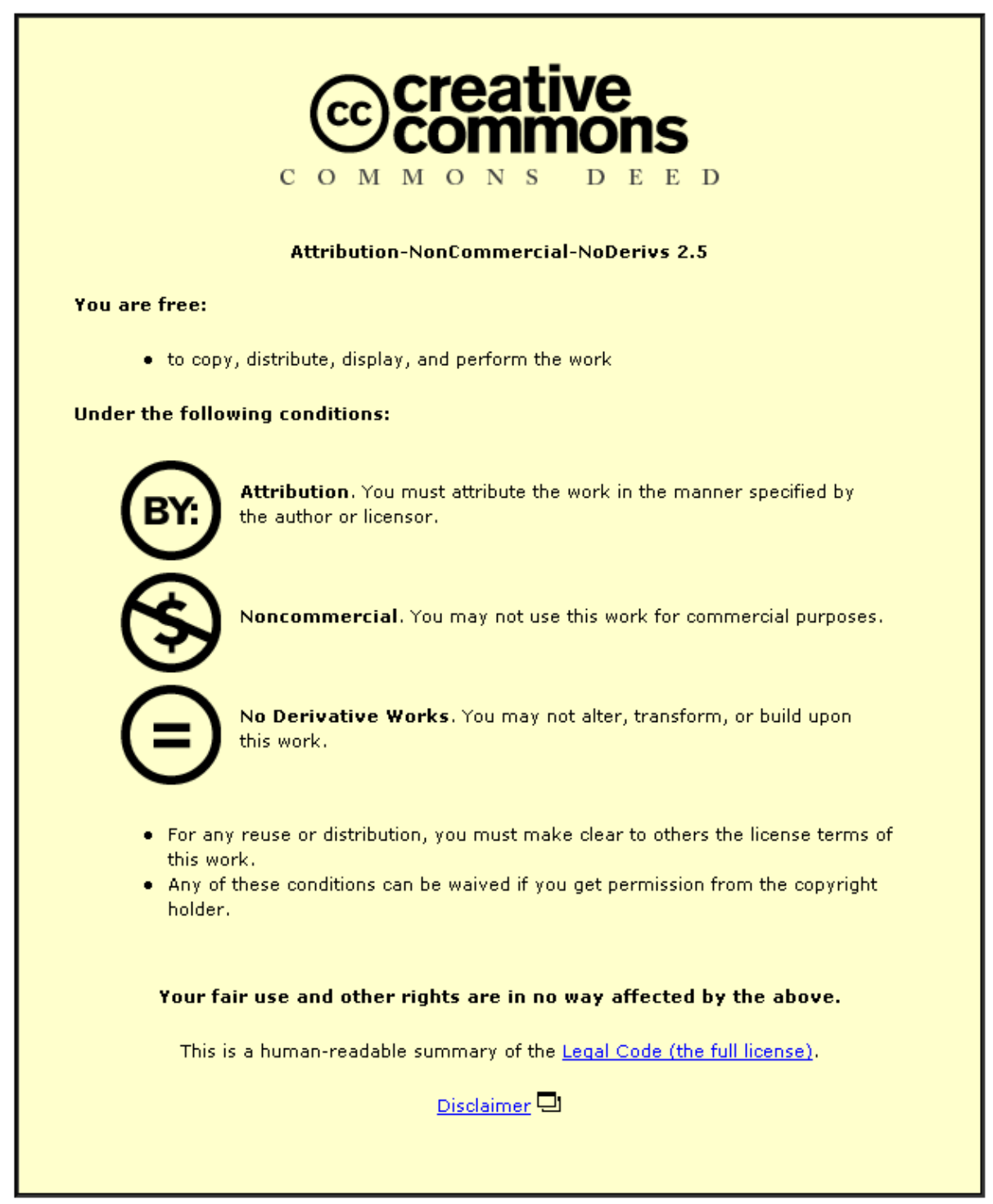

For the full text of this licence, please go to: http://creativecommons.org/licenses/by-nc-nd/2.5/ 


\title{
$\phi$-array: A novel method for fitness visualization and decision making in evolutionary design optimization
}

\author{
Monjur Mourshed ${ }^{\mathrm{a}, *}$, Shariful Shikder ${ }^{\mathrm{a}}$, Andrew D. F. Price ${ }^{\mathrm{a}}$ \\ ${ }^{a}$ Department of Civil and Building Engineering, Loughborough University, Loughborough, Leicestershire, LE11 3TU, United \\ Kingdom
}

\begin{abstract}
There is a growing interest in integrating model based evolutionary optimization in engineering design decision making for effective search of the solution space. Most applications of evolutionary optimization are concerned with the search for optimal solutions satisfying pre-defined constraints while minimizing or maximizing desired goals. A few have explored post-optimization decision making using concepts such as Pareto optimality, but mostly in multiobjective problems. Sub-optimal solutions are usually discarded and do not contribute to decision making after optimization runs. However, the discarded 'inferior' solutions and their fitness contain useful information about underlying sensitivities of the system and can play an important role in creative decision making. The need for understanding the underlying system behavior is more pronounced in cases where variations in the genotype space can cause non-deterministic changes in either the fitness or phenotype space and where fitness evaluations are computationally expensive.

The optimized design of an artificial lighting environment of a senior living room is used as a test case to demonstrate the need for and application of fitness visualization in genotype and phenotype spaces for effective decision making. Sub-optimal solutions are retained during optimization and visualized along with the optimum solution in a fitness array visualization system called $\phi$-array, developed as part of this research. The optimization environment is based on genetic algorithm (GA) in which a compute-intensive raytracing rendering engine, RADIANCE, is used to evaluate the fitness of prospective design solutions. Apart from describing the development of the optimization system and demonstrating the utility of $\phi$-array in effective decision making, this article explores optimization parameters and their effectiveness for artificial lighting design problems and the nature of their rugged fitness and constraint landscapes.
\end{abstract}

Keywords: Evolutionary optimization, $\phi$-array, Fitness visualization, Design exploration, Decision making, Artificial lighting

\section{Introduction}

There is a growing interest in the application of optimization techniques in engineering design that includes but not limited to, performance based design and operation of buildings. Design solutions are usually evaluated using simulation programs and the optimization algorithm provides a means to traverse the fitness landscape while satisfying predefined constraints. A wide range of problems has been addressed using model-based optimization in the built environment; e.g., thermal comfort and energy consumption [1]; selection of heating and cooling systems [2]; optimized controls for ventilation and/or

\footnotetext{
* Corresponding author

Email address: m.m.mourshed@lboro.ac.uk (Monjur Mourshed)

URL: http://monjur.mourshed.org/ (Monjur Mourshed)
} 
HVAC systems [3-6]; structural design [7-9]; outdoor thermal environment [10]; space layout planning [11]; building fenestration and envelope [12, 13]; and lighting design [14-16]. The models or underlying representations of problem domains, in these research, varied from hard-coded generalized equations of the system (e.g., [14]) resulting in fast evaluations to computationally expensive simulation programs such as RADIANCE (e.g., [15]), a physically-based raytracing rendering engine that often takes several minutes to hours to evaluate a single solution.

Evolutionary algorithms (EA) are well suited to building related problems due to the fact that fitness landscapes in most of these problems are rugged which can be effectively traversed by population based algorithms such as EAs. A comparative study of five evolutionary-based optimization algorithms can be found in [17]. Previous applications in the built environment and related domains have mostly been concerned with the search for optimal solutions satisfying predefined constraints while minimizing or maximizing desired goals. Most single objective optimizations admit only the 'perfect' or 'Utopian' solutions [18] and discard sub-optimal ones which usually do not contribute to decision making or the extraction of knowledge about the behavior of the underlying system. The same is also true for multiobjective problems that are re-formulated as single-objective prior to optimization; e.g., by applying scalar weights. A few have explored post-optimization decision making using concepts such as Pareto optimality in multiobjective problems [19-21]. However, Pareto-optimality usually involves the choice of single compromise solution from the non-dominated set according to design preferences [18]. Solutions that are not on the non-dominated Pareto front are often excluded from the decision making process.

However, the discarded 'inferior' solutions contain useful information about the underlying sensitivities of the system and can play an important role in the creative design process. Optimization is often applied on a small subset of the whole design problem because of: the limitations in modeling; inherent complexity; and discipline fragmentation. The optimal solution of the component problem, therefore, may not necessarily be the optimum for the whole. The excluded sub-optimal solutions, in particular those near the optimum in the solution space, can enable the consideration of other design goals that are not part of the optimization (e.g., aesthetics) while contributing to the creative aspects of problem solving such as design exploration. The need for understanding the underlying system behavior is more pronounced in cases where:

- Variations in the genotype space result in non-deterministic changes in either the fitness or phenotype space;

- The behavior of the system is not fully known, a-priori; and

- Fitness evaluations are computationally expensive.

To integrate sub-optimal solutions in the creative decision making process, a visualization system 
called $\phi$-array ( $\phi=$ adjusted fitness) has been developed in this research. The development of $\phi$-array took inspirations from molecular biology where the use of microarray assays are ubiquitous for visualizing large datasets in genomic analysis [22]. Microarray assays consist of arrayed series of spots of DNA oligonucleotides (or antibodies) each containing a specific DNA sequence and are considered to be one of the most important platforms for research in functional genomics. In simple terms, $\phi$-array is a form of multi-dimensioned data arrays, analogous with three-dimensional array-maps or pseudo-maps where each point represents associated $z$-value for the corresponding $x$ - and $y$-coordinates of a point. In a $\phi$ array map, the $x-y$ plane represents the genotype or phenotype space with the corresponding fitness as $z$-values.

To test the effectiveness of $\phi$-array in informed decision-making, a model-based evolutionary optimization environment has been developed for optimized design of artificial lighting in buildings. The domain of lighting design has been chosen as an application area because of:

- The lack of detailed knowledge about the underlying system behavior, mainly due to the absence of previous research on the nature of solution landscapes;

- The computationally expensive nature of fitness evaluations; and

- The potential for contribution to knowledge through the application of model-based optimization in the domain which is increasingly being considered as vital for enhancing occupant wellbeing.

The rest of the article is organized as follows. The design of artificial lighting in buildings is discussed with reference to the evolving design goals resulting from our improved understanding of the impact of lighting on humans. The particular design problem investigated here is discussed next along with the characteristics of design parameters, followed by a discussion on the optimization approach. The selection of optimization parameters are discussed by contextualizing them with a partial solution landscape, obtained by running brute-force simulations. The development of $\phi$-array is discussed next as well as its usefulness while analyzing optimization results. The article concludes with a summary of findings and directions for future research.

\section{Artificial lighting in buildings}

The design of an artificial lighting environment is a challenging task that involves the satisfaction of several physical and psychological criteria [23-25]. For example, an adequate level of illuminance over the working plane needs to be provided while maintaining the uniformity of illumination; bright patches need to be avoided to eliminate or reduce glare and visual discomfort; etc. Additional criteria need to be satisfied for specialized lighting design; e.g., designs for people with low vision such as the elderly [26], adding another layer of complexity. The trade-off between lighting design criteria is not 
always straightforward. The distribution of light is affected by luminaire characteristics; interior surface geometry, orientation and reflectance; and interior object geometry and reflectance. Minor changes in the location of a luminaire in a given space can have a significant impact on one or more of the lighting design criteria, because of the reflection and re-reflection of light off various interior surfaces. The result of such interdependent interactions is usually a rugged solution landscape, which is non-deterministic in nature.

The search for an optimum design satisfying multiple criteria in a rugged solution space is time consuming if brute-force methods are applied and physically-based lighting simulation programs (e.g., RADIANCE [27]) are used for the evaluation of proposed solutions. Alternatively, simplified methods such as the average illuminance method [28] can be used but the results are not always within acceptable limits of uncertainty. Despite its usefulness in achieving better performance, exploration is seldom used as a design technique in conventional design methods. Conventional approaches to lighting design are usually limited only to the appraisal of a proposed solution. In most cases, simulation tools or simplified methods are used merely to confirm or reject designers' assumptions, mainly because of the lack of an effective search mechanism. Potential solutions with better performance thus remain unexplored. Therefore, there is a need to integrate effective search and optimization techniques with design evaluations to achieve better performance.

Most model-based optimizations in lighting design were concerned with the degree of satisfaction of the level of illuminance required for visual tasks. Fewer studies have been conducted on the optimization of artificial lighting design, compared to daylighting and its impact on building energy consumption. Health and safety aspects that requires simultaneous optimization of both horizontal and vertical illuminance levels and their distributions in interior spaces as well as the prevention of visual discomfort have not been addressed adequately in previous research. The solution landscape for vertical illuminance is rugged with an element of randomness in its distribution - making it difficult to solve, numerically.

To address the challenge in traversing the rugged landscapes in lighting design, an evolutionary optimization environment has been developed, consisting of a genetic algorithm (GA) for an effective search of the solution space. For a realistic representation of the problem domain health and safety aspects of illumination are considered in the design criteria, in addition to the physical aspects. Luminaire types and their geometric locations are considered as design variables. Design requirements such as illuminance and luminance thresholds are modeled as constraints whereas the maximization of the uniformity of illumination is modeled as a goal or objective. 


\section{Problem definition}

A senior living room has been selected as the problem case in this study. The internal spatial configuration and furniture layout of the room is illustrated in Figure 1 and 2. The bathroom protrudes into the living area and results in an L-shaped spatial configuration. The space near the entrance door poses a particular challenge in lighting design due to the proportion of the space (entry area) and the close proximity of the enclosing surfaces. The irregular shape of the room adds more complexity to lighting design in meeting the desired illuminance uniformity, compared to a purely rectangular room. The adoption of a realistic design problem with furniture and fixtures as a test case sets this study apart from previous research that were mostly based on non-realistic ideal scenarios. Conventional wisdom suggests a two-luminaire solution to the problem for providing adequate illuminance in the entry area while maintaining a level of illuminance uniformity in the entire space. The problem case, therefore, adopts a two-luminaire approach to lighting design.

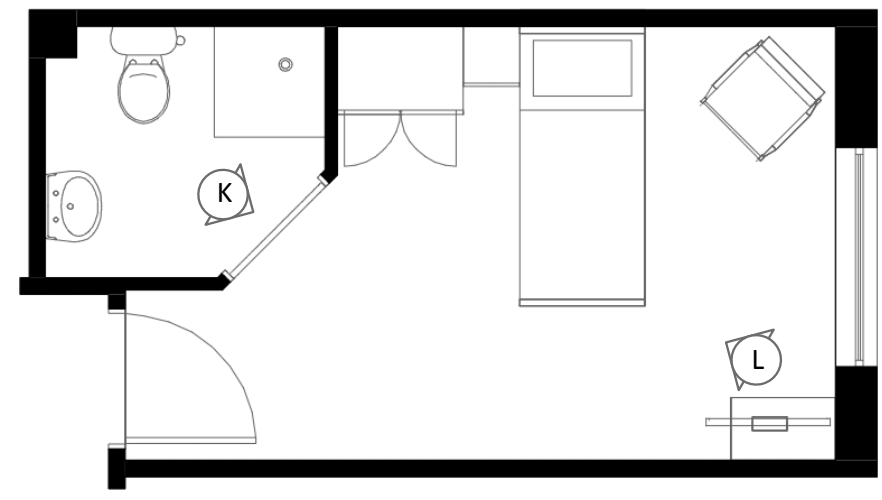

Figure 1: Plan of the senior living room showing furniture layout and directions of axonometric views.

The selection of luminaires depends on the technical aspects of lighting design and the target lighting quality, which can be obtained by selecting appropriate luminaires configured with appropriate lamps and intensity distribution profiles. The Chartered Institution of Building Services Engineers (CIBSE) [28] suggests that the selection of luminaires should respect architectural quality of the space, in addition to the desired performance criteria. For spaces occupied by seniors, the Illuminating Engineering Society of North America (IESNA) [23] recommends luminaires with lamps having a correlated color temperature (CCT) between 3500 and $4000 \mathrm{~K}$. Sufficient color rendition is also required to ensure adequate discrimination between colors. To meet these criteria, lighting installations with compact fluorescent lamps (CFL) were selected with a CCT of $4000 \mathrm{~K}$ and a color reddening index of 85.

The ceiling of the problem case is suspended from the permanent roof/ceiling above and constructed out of ceiling tiles of size $600 \times 600 \mathrm{~mm}$. To respect the architectural qualities of the space, recessed mod- 


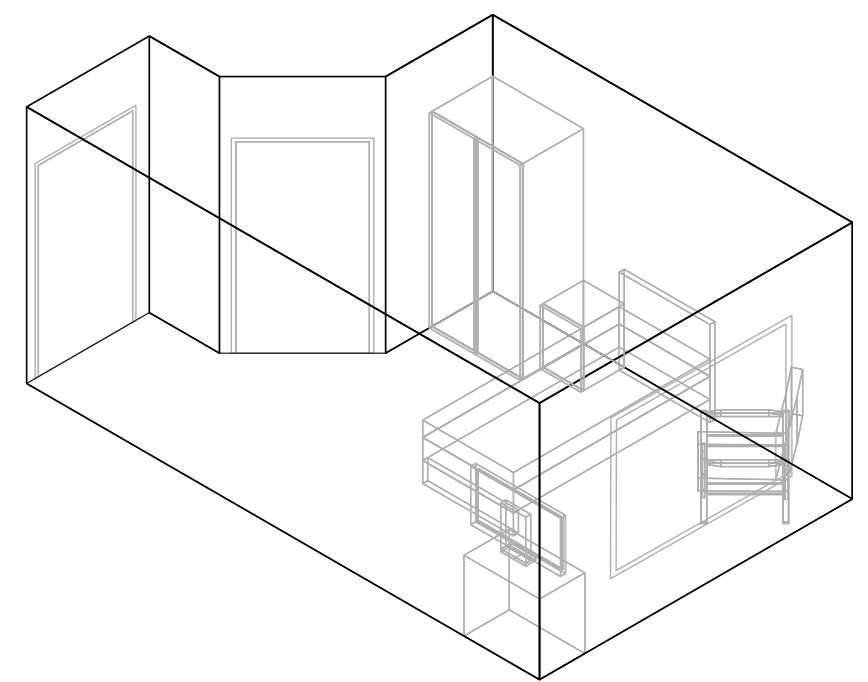

Figure 2: 3D wireframe view of the model of the senior living room.

ular luminaires have been considered. Subsequently, six CFL luminaires were selected for this investigation. A combination of two luminaires out of the selected six was expected to formulate the desired solution. Luminous flux of the selected luminaires ranged from 1200 to $7000 \mathrm{~lm}$. Among the luminaires, three were of rectangular shape (size: $600 \times 600 \mathrm{~mm}$ ) and named as primary luminaire type, $T_{\mathrm{p}}$. The remaining three were of radial shape (diameter: $200 \mathrm{~mm}$ ) and named as secondary luminaire type, $T_{\mathrm{s}}$. Due to the smaller size of the radial shaped luminaires, their position in the ceiling tile was not fixed to the centre of the tile. Rectangular shaped luminaires, however, could only occupy the full size of the ceiling tile. The position grids for the primary and secondary luminaire types are illustrated in Figure 3.

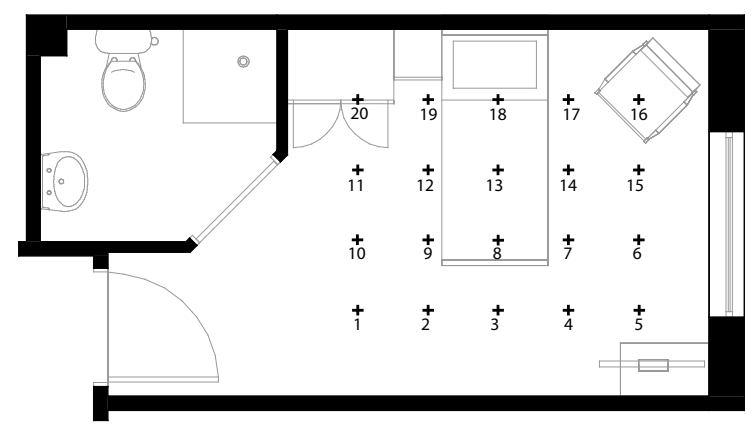

(a) $T_{\mathrm{p}}$

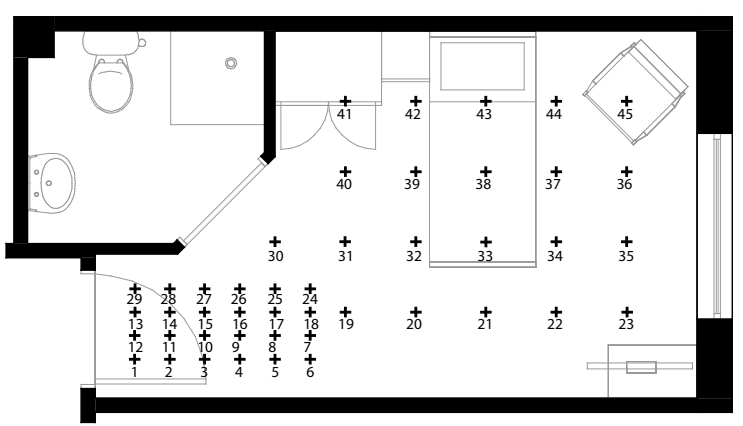

(b) $T_{\mathrm{s}}$

Figure 3: The position grids for primary $\left(T_{\mathrm{p}}\right)$ and secondary $\left(T_{\mathrm{s}}\right)$ luminaire types. 
Table 1: Photometry and attributes for primary luminaire types, $T_{\mathrm{p}}$.

\begin{tabular}{|c|c|c|}
\hline$T_{\mathrm{p}}$ Photometry & Attribute & Value \\
\hline \multirow{4}{*}{ A } & Lamp & $\mathrm{T} 26 / 18 \mathrm{~W}$ \\
\hline & Number & 3 \\
\hline & Total lum. flux & $4050 \mathrm{~lm}$ \\
\hline & Total power & $58 \mathrm{~W}$ \\
\hline \multirow{4}{*}{ B } & Lamp & $\mathrm{T} 26 / 18 \mathrm{~W}$ \\
\hline & Number & 4 \\
\hline & Total lum. flux & $5400 \mathrm{~lm}$ \\
\hline & Total power & $74 \mathrm{~W}$ \\
\hline \multirow{4}{*}{ C } & Lamp & $\mathrm{T} 16 / 24 \mathrm{~W}$ \\
\hline & Number & 4 \\
\hline & Total lum. flux & $7000 \mathrm{~lm}$ \\
\hline & Total power & $99.2 \mathrm{~W}$ \\
\hline
\end{tabular}

\subsection{Design criteria}

A critical review of literature [29] identified that with increased age, the elderly suffer with obvious optical changes, causing one or more visual diseases such as cataract, diabetic retinopathy, glaucoma, etc. Suffering with one or more of these diseases result in various visual difficulties; e.g., increased amount of scattering of retinal illumination, reduced contrast sensitivity and slowed light-dark adaptation, etc. [30]. To offset these visual difficulties further criteria needs to be met for the design of lighting and the visual environment for the elderly. Recommendations suggest that the elderly require increased amount of illumination to perform equally as a younger adult. In addition to providing adequate levels of horizontal and vertical illuminations, the distribution of illumination over reference planes and luminance contrast of the key surfaces need to be considered carefully. Industry standards and guidance on lighting and low vision were consulted for recommended ranges of illumination level and its distribution. Design criteria for the elderly and low vision were obtained from the guidance on low vision, published by IESNA [23, 26] and the Commission Internationale de l'Éclairage (CIE) [31]. CIBSE Code for Lighting [25] was consulted for information pertaining to the general lighting design criteria. For further guidance on the lighting design of health spaces, the CIBSE Lighting Guide 2 [32] was consulted. 
Table 2: Photometry and attributes for secondary luminaire types, $T_{\mathrm{s}}$.

\begin{tabular}{lll}
\hline$T_{\mathrm{s}}$ Photometry & Attribute & Value \\
\hline & $\begin{array}{l}\text { Lamp } \\
\text { Number }\end{array}$ & 1 \\
& Total lum. flux & $1200 \mathrm{~lm}$ \\
Total power & $20.5 \mathrm{~W}$ \\
\hline & Lamp & TC-DEL/18 W \\
& Number & 2 \\
& Total lum. flux & $2400 \mathrm{~lm}$ \\
& Total power & $40 \mathrm{~W}$ \\
\hline & Lamp & TC-TEL/26 W \\
& Number & 2 \\
& Total lum. flux & $3600 \mathrm{~lm}$ \\
& Total power & $51 \mathrm{~W}$
\end{tabular}

The goal of the design optimization activity was the maximization of horizontal illuminance uniformity, $U_{\mathrm{h}}$, to ensure uniform light over working areas. This is because of the fact that rapid changes of illumination can be dangerous, particularly for the partially-sighted as their adaptation time is higher and cause visual disability when moving from a brightly- to a dimly-lit area [31].

The optimization was subjected to several constraints. To ensure adequate light over the working plane, the mean horizontal illuminance, $E_{\mathrm{h} \text {,mean }}$, was set to be higher than $300 \mathrm{~lx}$ [26]. For easy identification of the exit and door handle, mean vertical illuminance on the door, $E_{\mathrm{d} \text {,mean }}$, was set to be higher than $100 \mathrm{~lx}$. This is due to the fact that a level of vertical illumination needs to be maintained for distant objects and surfaces with text or graphic symbols so that the partially sighted and demented patients can orient themselves in the space and identify objects. The limiting value of $100 \mathrm{~lx}$ on wall and door surfaces was based on UK building regulations [33]. A limit of $750 \mathrm{~lx}$ on the maximum horizontal illuminance, $E_{\mathrm{h}, \max }$, was set to avoid bright patches or luminance spots; i.e., to avoid undue glare to the recumbent or ambulatory occupant in the senior living room.

The amount of light falling onto a surface creates the visible luminance with the combination of surface color and texture quality. Vertical illuminance is, therefore, responsible for creating the luminance 
of the wall and door surfaces. Luminance within the visual angle is significant in interior lighting design due to its direct association with glare. Two principal reasons for occurring glare are: too much light falling on the eye; and the range of luminances being too high within the visual angle. It is thus necessary to control surface luminances within the visual angle to limit glare. To avoid any excessive luminance in vertical surfaces by the luminaires, the maximum spot luminance, $L_{\mathrm{v}}$, max , was set to be below $1500 \mathrm{~cd} / \mathrm{m}^{2}$ [32]. Maximum vertical illuminance, $E_{\mathrm{v}, \max }$, on any point on the vertical surface was used as a proxy for $L_{\mathrm{v}, \max }$, which was obtained using Equation 3 . The wall surfaces in this study are considered to be uniformly diffused with a reflectance $(\rho)$ of 0.7 .

\subsection{Calculation procedures}

Lighting calculations are initially performed in RADIANCE, a backward raytracing simulation engine. Horizontal and vertical analysis grids, as shown in Figure 4, were set up to obtain illuminance values. The horizontal grid contains 78 sensor points at $0.75 \mathrm{~m}$ height from the floor. The vertical analysis grid covering all visible wall and door surfaces consists of 685 points in total. The values of the objective and constraints were obtained by post-processing outputs from RADIANCE simulation using the calculation procedures described below.
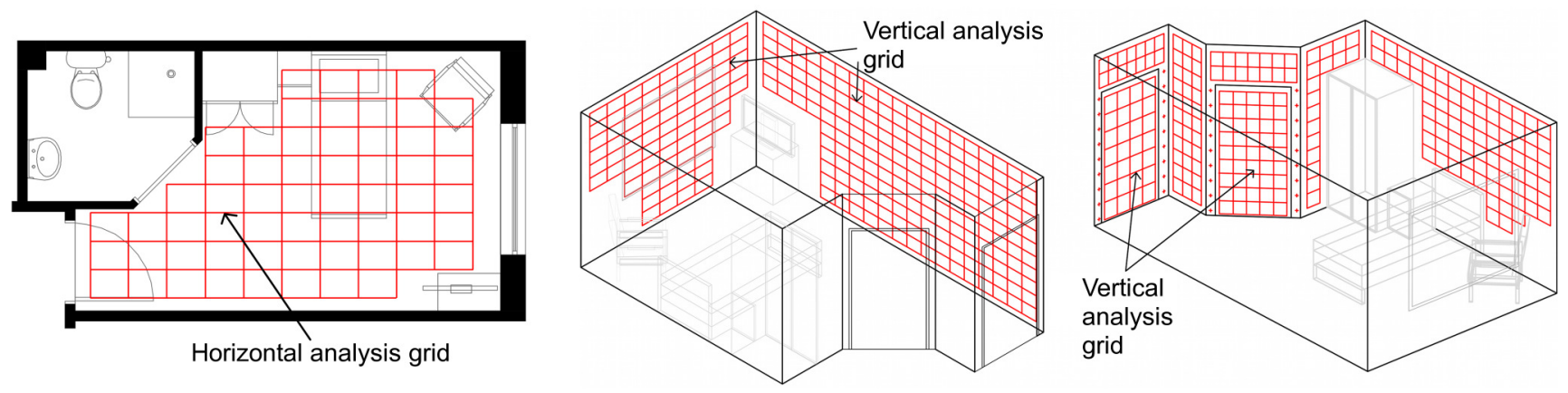

Figure 4: Horizontal and vertical analysis grids.

The mean horizontal illuminance in the room was calculated using the Equation 1.

$$
E_{\mathrm{h}, \text { mean }}=\sum_{i=1}^{n} \frac{E_{\mathrm{h}, i}}{N_{\mathrm{t}}}
$$

where, $E_{\mathrm{h} \text {,mean }}$ is the mean horizontal illuminance (lx) over the reference plane, $E_{\mathrm{h}, i}$ is the horizontal illuminance (lx) at the reference point $i$ and $N_{\mathrm{t}}$ is the total number of reference points.

The horizontal illuminance uniformity over the reference plane, expressed as the ratio of minimum to mean illuminance, was calculated using the Equation 2 .

$$
U_{\mathrm{h}}=\frac{E_{\mathrm{h}, \text { min }}}{E_{\mathrm{h}, \text { mean }}}
$$


where, $U_{\mathrm{h}}$ is the horizontal illuminance uniformity over the reference plane, $E_{\mathrm{h} \text {,min }}$ is the minimum horizontal illuminance (lx) and $E_{\mathrm{h} \text {,mean }}$ is the mean horizontal illuminance (lx).

The limiting illuminance on a vertical surface was obtained from the Equation 3.

$$
E_{\mathrm{v}, \max }=\frac{L_{\mathrm{v}, \max } \times \pi}{\rho}
$$

where, $L_{\mathrm{v}, \max }$ is the maximum luminance at the reference point $\left(\mathrm{cd} / \mathrm{m}^{2}\right)$ on a vertical surface, $E_{\mathrm{v}, \max }$ is the maximum illuminance at the reference point (lx) and $\rho$ is the reflectance of the surface (-).

\section{Optimization approach}

The optimization of luminaire positions is defined as single-objective constrained optimization and given by:

minimize:

$$
f(\mathbf{x})=-U_{\mathrm{h}}
$$

subject to:

$$
\begin{aligned}
E_{\mathrm{h}, \text { mean }} & \geq 300 \\
E_{\mathrm{h}, \text { max }} & \leq 750 \\
E_{\mathrm{d}, \text { mean }} & \geq 100 \\
E_{\mathrm{v}, \text { max }} & \leq 1500
\end{aligned}
$$

where, $\mathrm{x}$ is a vector of discrete variables of primary and secondary luminaire types and positions:

$$
\mathbf{x}=\left[T_{\mathrm{p}}, T_{\mathrm{s}}, P_{\mathrm{p}}, P_{\mathrm{s}}\right]^{T}
$$

where, $T_{\mathrm{p}}$ is the primary luminaire type, $T_{\mathrm{s}}$ is the secondary luminaire type, $P_{\mathrm{p}}$ is the primary luminaire position and $P_{\mathrm{s}}$ is the secondary luminaire position.

\subsection{Constraint handling}

GAs are naturally an unconstrained optimization algorithm and its success depends on the evaluation of fitness of the individuals in a population. Formulating a constrained optimization as an unconstrained GA; i.e., incorporating constraint violation in the fitness is challenging [34, 35]. Several methods have been proposed and successfully utilized in constrained GA applications. A review of the methods can be found in [34]. Among these methods, the penalty function method [36] is used widely. The penalty function method, adopted in this study, is given by: 


$$
\phi(\mathbf{x})=f(\mathbf{x})+\sum_{i=1}^{n} r_{i} G_{i}
$$

where, $\phi(\mathbf{x})$ is the new objective function to be optimized after adding penalty scores to $f(\mathbf{x}), r_{i}$ is the penalty parameter for the constraint function $i$, and $G_{i}$ is the inequality constraint function.

\subsection{Fitness and constraint landscapes}

A brute-force parametric simulation of the partial solution space was carried out to investigate the characteristics of the fitness and constraint landscapes. A combination of two luminaire types, B and N, were used and their respective positions were allowed to vary between the lower and upper bounds of the variables, $P_{\mathrm{p}}$ and $P_{\mathrm{s}}$. The resulting partial solution space, representing $11.1 \%$ of the total solution space, is shown in Figure 5 where design criteria are plotted against the positions of the luminaires.

Figure 5a shows the solution or fitness landscape; i.e., illuminance uniformity over the horizontal reference plane, $U_{\mathrm{h}}$. The landscape is non-linear and rugged with abundant local maxima and minima. The ruggedness of the landscape is more pronounced than previous parametric explorations of design solution spaces involving mainly thermal design parameters such as building form and orientation [37], glazing vs wall area ratio [12], etc. The room and furniture layouts along with varying surface reflectances have an impact on the level of illuminance at a particular point on the reference plane and can register gentle variations in the phenotypic representation, which can be attributed as a reason for the differences in landscape characteristics.

Figure $5 \mathrm{~b}$ shows mean illuminance on the horizontal reference plane, $E_{\mathrm{h}, \text { mean }}$. The trend for $E_{\mathrm{h} \text {,mean }}$ appears to be uniform except at a few grid positions where the value for mean illuminance drops suddenly. This happens when both luminaires are located very close to each other or on top of each other. Figure $5 \mathrm{c}$ illustrates maximum illuminance on vertical wall surfaces, $E_{\mathrm{v}, \max }$, which appears to be consistently high for a few specific combinations of luminaire positions. Figure 5d illustrates mean illuminance on the entry door, $E_{\mathrm{d} \text {,mean }}$. The peaks demonstrate that particular combinations of luminaires are suitable for maximizing illuminance on the door. Both vertical illuminance indices, $E_{\mathrm{v}, \text { max }}$ and $E_{\mathrm{d} \text {,mean }}$ varies significantly for different luminaire positions, which explain the difficulty in providing a uniform illumination over vertical surfaces.

The brute-force study identified the varying characteristics of lighting design parameters and their landscapes. Differences exist between vertical and horizontal illuminance landscapes. For minor changes in the design parameters; i.e., the positions of luminaires, there were significant changes in vertical illuminance of an investigated interior surface. It is evident that the conventional trial and error approach of finding better solutions is insufficient. Advanced search methods such as optimization, therefore, need to be employed for maximizing the efficiency of lighting systems in providing a safe and comfortable environment for its occupants. 


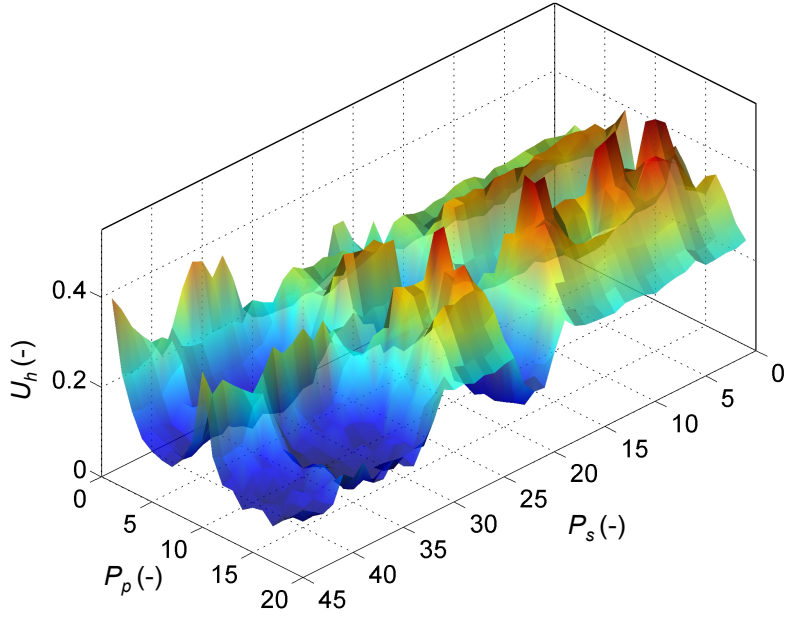

(a) $U_{\mathrm{h}}$

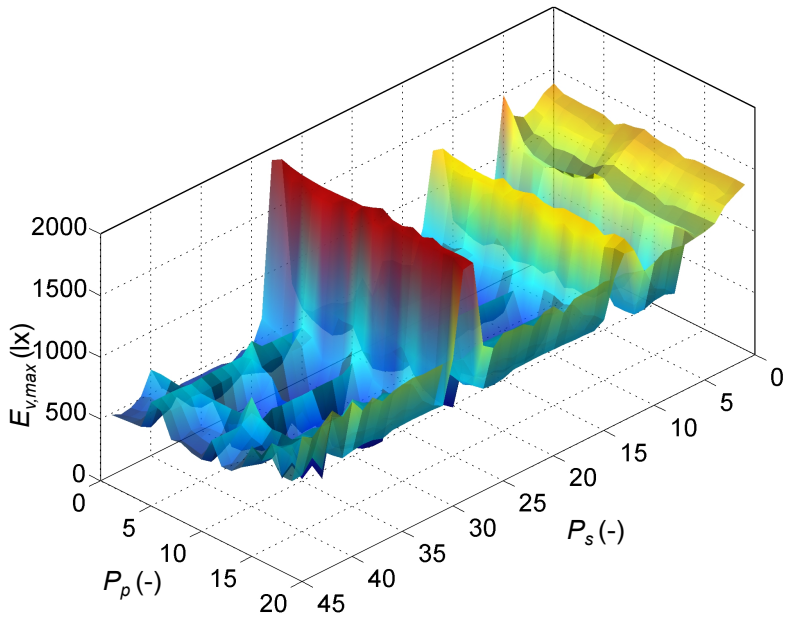

(c) $E_{\mathrm{v}, \max }$

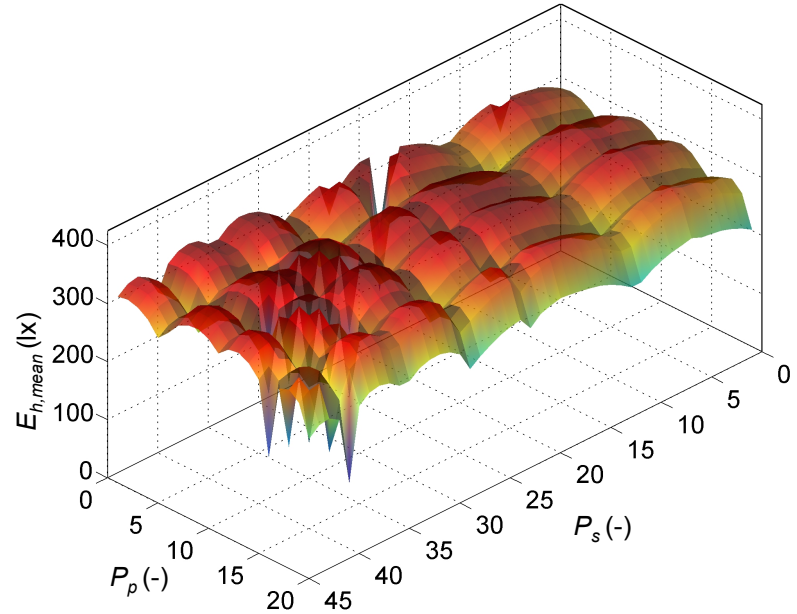

(b) $E_{\mathrm{h}, \text { mean }}$

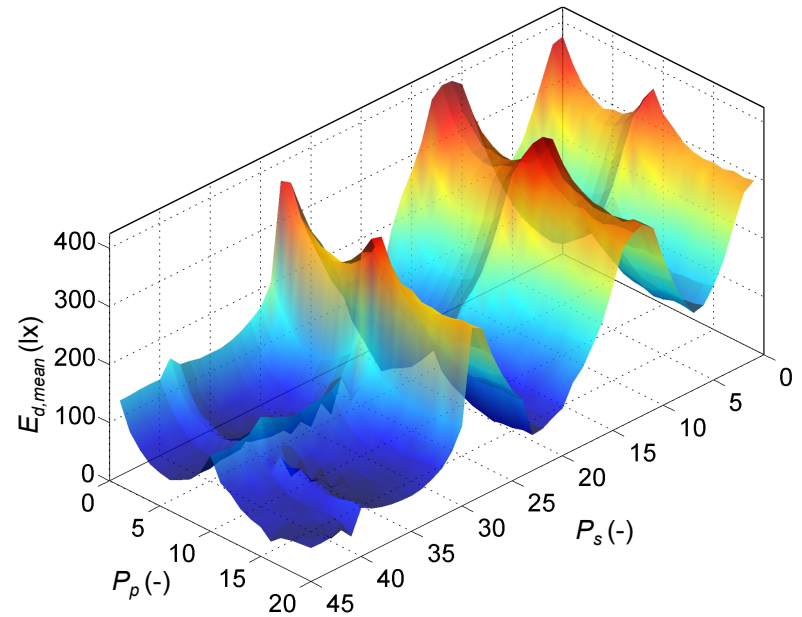

(d) $E_{\mathrm{d} \text {,mean }}$

Figure 5: The fitness (a) and constraint landscapes (b-d) for the combination of luminaire types B and N: (a) illuminance uniformity over the horizontal reference plane, $U_{\mathrm{h}}=-f(\mathrm{x})$; (b) mean illuminance over the horizontal reference plane, $E_{\mathrm{h} \text {,mean }}$; (c) maximum illuminance on vertical wall surfaces, $E_{\mathrm{v} \text {,max }}$; and (d) mean illuminance on the entrance door, $E_{\mathrm{d} \text {,mean }}$.

\subsection{Optimization algorithm}

A real-number encoded genetic algorithm (GA) was used in this study. Values were scaled to nearest integers within the specified lower and upper bounds during initialization and mutation. The following GA strategies were employed:

- Fixed initialization of population was used for the crossover probability study and random initialization was used for the final optimization; 
- Selection of individuals in a population for genetic operations is based on tournament selection with rank based fitness scaling;

- Fitness scaling is done by ranking raw fitness scores and the rank of an individual is its position in the sorted scores;

- Elitism is employed in the form that the single best solution from the previous generation is preserved in the new generation;

- The GA stops when the specified maximum number of generations is reached or when the weighted average change in the fitness function value over stalled generations is less than the specified function tolerance; and

- The fitness of each evaluated individual is stored to save computation time spent on simulation.

Population size for real-encoded genetic algorithms is usually determined by ad-hoc approaches and depends on a researcher's experience [38] of the type of the problem and the solution landscape. The choice of population size has been reported to cause the runtime of the GA to vary by several orders of magnitude [39]. The compute-intensive nature of the physically-based simulation ${ }^{1}$ in this study meant that the GA's population size needed to be carefully chosen to reduce overall runtime but not sacrificing the speed of convergence. Previous studies have found that a small population is quite effective [40] when the dimension of the problem is small [41] and the GA is allowed to run for many generations [39]. The population size for the GA in this study was, therefore, chosen to be 20 . The crossover probability, $P_{\mathrm{c}}$, was set to 0.4 , which was based on a parametric study, described in the following section. The mutation probability, $P_{\mathrm{m}}$, was, therefore, $0.6\left(P_{\mathrm{m}}=1-P_{\mathrm{c}}\right)$. The maximum number of generations was set to 100 as one of the stopping criteria.

\subsection{Crossover probability}

The quality of solutions found in a GA run is closely related with the genetic diversity of the population with time, the lack of which may result in premature convergence to local optima of the objective function [42]. Diversity of the population is expressed in terms of the average distance between individuals and is considered high when the average distance between the individuals is large. The parameters that affect the diversity and ultimately the premature convergence are: population size; and mutation and crossover probabilities [43]. Because of the rugged solution landscape in this study, as illustrated in Figure $5 \mathrm{a}$, the mutation probability needs to be carefully chosen to maintain genetic diversity; i.e., there needs to be a balance between the rates of crossover and mutation.

\footnotetext{
${ }^{1}$ Each function or simulation call takes approximately 15 minutes to complete.
} 
An experiment was set up to determine a suitable value for crossover probability, $P_{\mathrm{c}}$. A limit on the number of generations $\left(N_{\mathrm{g}}=40\right)$ was set while $P_{\mathrm{c}}$ was allowed to vary between 0 and 1 , with a step size of 0.05 . The reason for setting a lower limit for the number of generations was to investigate the speed of convergence against the quality of the solution found. The experiment was run for four times and the results were analyzed. Figure 6 shows the best fitness, $\phi^{*}(\mathbf{x})$ against different values of $P_{\mathrm{c}}$, along with a quadratic polynomial curve fit of the data. Both lower and higher values of $P_{\mathrm{c}}$ caused a decrease in the quality of the solution found. The performance appears to worsen for higher values of $P_{\mathrm{c}}$. A higher rate of mutation appears to be important for this problem type to maintain genetic diversity. $P_{\mathrm{c}}$, with values of 0.2 and 0.4 , seems to be consistently producing better solutions, before the maximum number of generations is reached. The value for $P_{\mathrm{c}}$ was, therefore, set to 0.4 for the final run to balance between mutation and crossover rates. The values for the sum of squares due to error (SSE), root mean squared error (RMSE), R-square and adjusted R-square of the goodness of fit data are provided in Table 3. Descriptive statistics of the data are presented in Table 4.

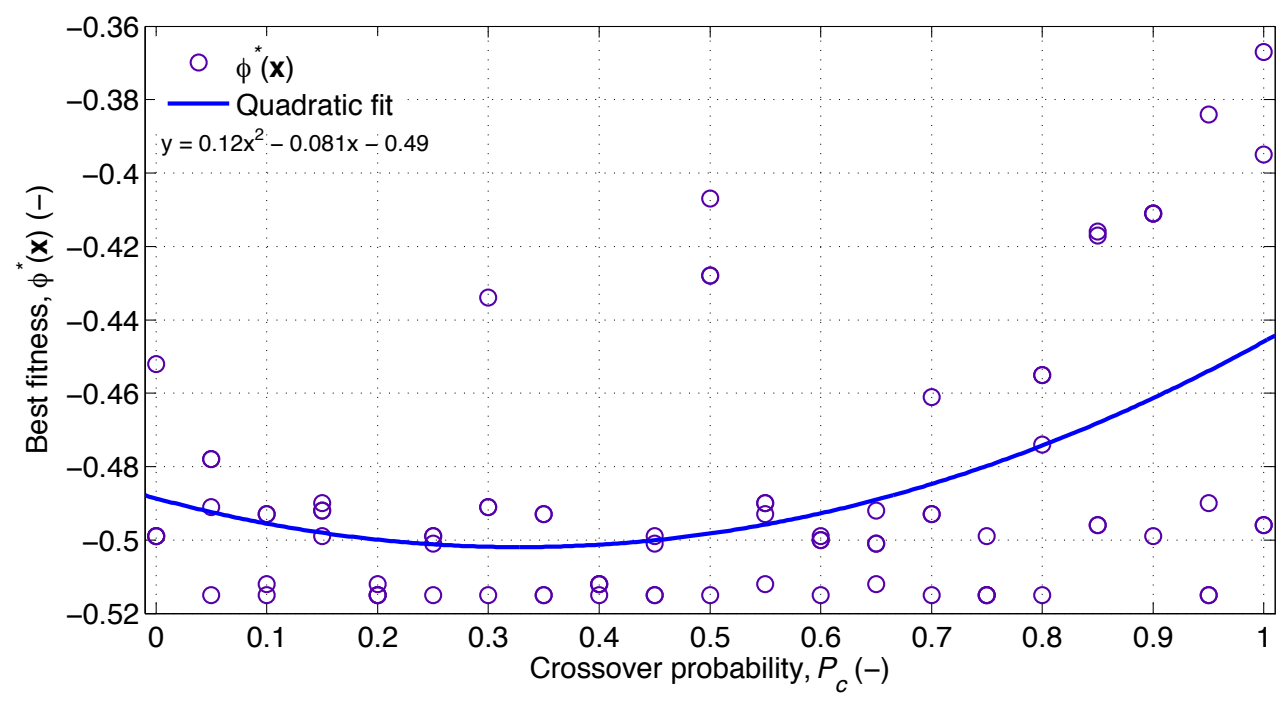

Figure 6: Best fitness, $\phi^{*}(\mathbf{x})$, achieved using different values of crossover probability, $P_{\mathrm{c}}$.

Table 3: Goodness of fit data for the quadratic fit; $\phi^{*}(\mathbf{x})$ vs. $P_{\mathrm{c}}$.

\begin{tabular}{lrrr}
\hline SSE & R-square & Adjusted R-square & RMSE \\
\hline 0.08268 & 0.215 & 0.1957 & 0.03195 \\
\hline
\end{tabular}


Table 4: Statistics on data from crossover probability study.

\begin{tabular}{lrrrrr}
\hline Min & Max & Mean & Median & SD & Range \\
\hline 0.367 & 0.515 & 0.4869 & 0.499 & 0.03562 & 0.148.
\end{tabular}

\section{Optimization results}

Figure 7 illustrates that the best fitness $\left(\phi^{*}(\mathbf{x})=-0.515\right)$ was achieved on the $23^{\text {rd }}$ generation. The total number of evaluations for the complete run was 2020 . However, the number of calls to the simulation engine, RADIANCE, was $1105^{2}$. The $45 \%$ savings on computation time was due to the use of memory in GA. The mean fitness curve illustrates that a diverse population was searched throughout the optimization run. This is mainly due to the adoption of a low crossover probability $\left(P_{\mathrm{c}}=0.4\right)$. Mutation was the dominant evolution strategy and enabled a wider search of the rugged solution landscape, in which the design vector, $\mathrm{x}$, comprised index variables. The solution metrics of the optimization run is given in Table 5, showing that the constraints were met.

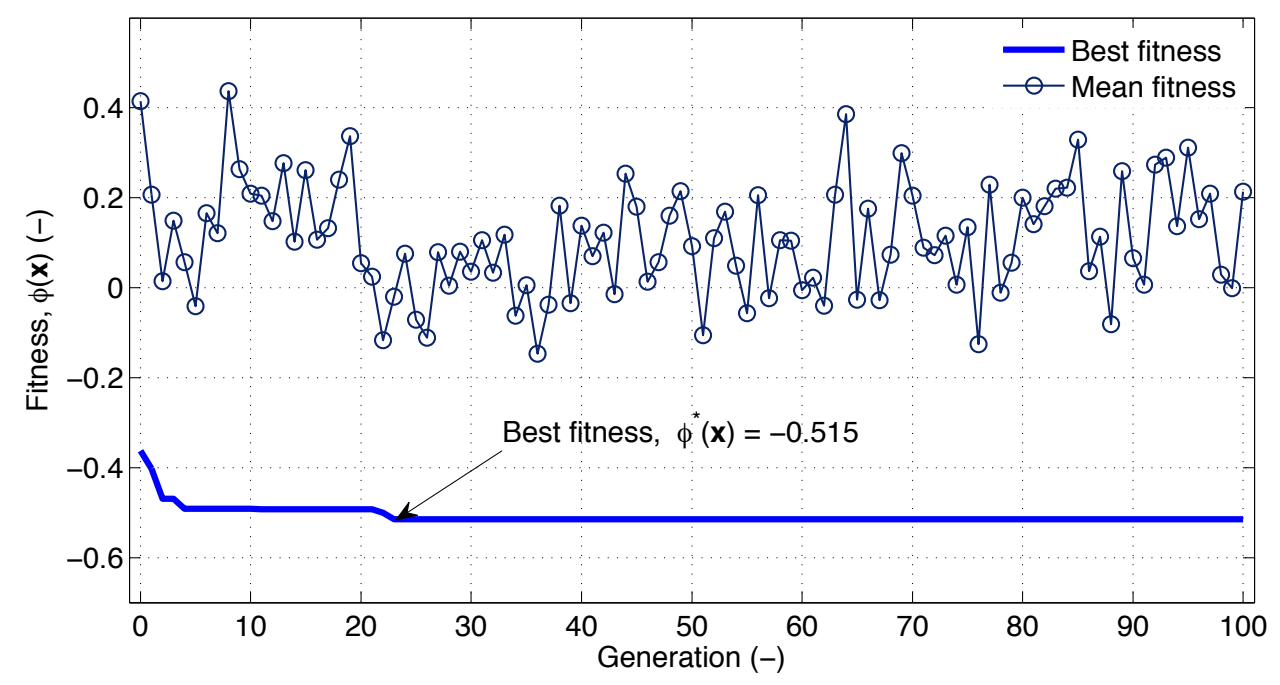

Figure 7: Best and mean fitnesses in each generation.

The illuminance distribution maps of the best design solution over horizontal and vertical reference planes are illustrated in Figure 8. The plan shows the optimal positions and types of primary and sec-

\footnotetext{
${ }^{2}$ The number of evaluations for 23 generations was: $(23+1) \times 20=480$, when the best fitness was achieved. However, the actual calls to the simulation engine would be much less because of the use of memory.
} 
Table 5: Solution metrics for the optimization.

\begin{tabular}{|c|c|c|c|c|c|c|c|c|}
\hline$T_{\mathrm{p}}$ & $T_{\mathrm{s}}$ & $P_{\mathrm{p}}$ & $P_{\mathrm{s}}$ & $U_{\mathrm{h}}$ & $E_{\mathrm{v}, \max }$ & $E_{\mathrm{h}, \text { mean }}$ & $E_{\mathrm{h}, \max }$ & $E_{\mathrm{d}, \text { mean }}$ \\
\hline \multicolumn{5}{|c|}{$(-)$} & \multicolumn{4}{|c|}{ (lx) } \\
\hline B & $\mathrm{N}$ & 14 & 17 & 0.515 & 387.7 & 337.7 & 549.46 & 157.9 \\
\hline
\end{tabular}

ondary luminaires, denoted by B and N. Conventional positioning of the primary and secondary luminaires is also shown in the figure and denoted by $\mathrm{B}^{\prime}$ and $\mathrm{N}^{\prime}$, respectively. The positions of $\mathrm{B}^{\prime}$ and $\mathrm{N}^{\prime}$ are usually the centers of gravity of the abstract rectangles created by the enclosing interior surfaces. The difference between the optimum and conventional positions of the luminaires is significant, in particular in the case of secondary luminaire above the entry space. This finding alone demonstrates the advantages of the optimal design process over conventional heuristics-based approach. Visualizing optimal solutions on its own (Figure 8) or in relation to optimization progress (Figure 7) may give indications of the algorithm's performance but hardly any information about the underlying behavior of the system. To overcome this limitation, the $\phi$-array method of visualization is applied to explore the genotype and phenotype spaces.
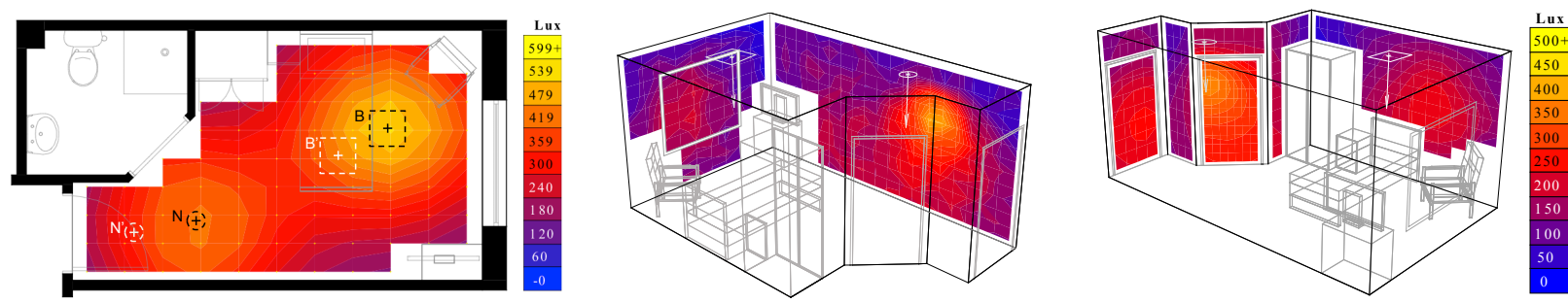

Figure 8: Illuminance maps over horizontal and vertical reference planes of the optimum design solution.

\section{6. $\phi$-array visualization}

The approach for generating a $\phi$-array is to code a set data that will enable the visualization of fitness of individual solutions in their genotype or phenotype space. In its simplest form, genotype or phenotype space can be represented by the $x-y$ plane with $z$-values representing fitness. In some cases, genotype and phenotype spaces can be interchanged in the $x-y$ plane if translations between genotypes and phenotypes are straightforward and if there is an agreement between the dimensions. The 3D array can be expanded by adding further dimensions such as constraint violation which can be represented using color or size of the individual fitness value in the $z$-axis. Other variations may include the normalization 
of the data (e.g., fitness) over a suitable range for visualizing heterogenous information.

Three $\phi$-arrays have been created in this research to demonstrate their effectiveness in extracting domain knowledge and in enabling design exploration. The phenotype space, same as the genotype space in this problem, is coded in the $x-y$ plane. Fitness values are visualized as varying unfilled scatter markers representing $z$-values in the map. Another dimension is added by changing the colors of individual scatters. In Figure 9, the $4^{\text {th }}$ dimension represents fitness in the phenotype space, same as the $3^{\text {rd }}$ dimension, but in reverse order. In Figures 11 and 12, the $4^{\text {th }}$ dimension represents violations of constraints and luminaire types. The $\phi$-array is versatile in the way it enables the visualization of fitness, constraints and variables in the same genotype/phenotype space while maintaining consistency in spatial representation. The consistency is quite important for orienting oneself in a decision space when the dimensionality of the problem exceeds what one can easily comprehend in a single map.

\subsection{Optimal vs. sub-optimal}

As a first step, the optimal solution is visualized along with sub-optimal ones in a phenotypic $\phi$-array in Figure 9. The phenotype space consists of two geometric variables, $P_{\mathrm{p}}$ and $P_{\mathrm{s}}$, the primary and secondary luminaire positions. The size of a marker gives an indication of its fitness; the bigger the marker, the better its fitness. The color of a marker maps to its fitness as well, but in reverse order: the lower the value in the colormap, the better the fitness of the corresponding individual. It appears that certain primary luminaire positions; i.e., $P_{\mathrm{p}}=7$ and 14 , are associated with most rows of designs that satisfied the constraints while minimizing fitness. Both positions are next to each other on the analysis grid (Figure 3a) and are equidistant from the centre of gravity of the grid. Constraints appear to have been violated (represented by warm-colored markers) for most positions of secondary luminaire $\left(P_{\mathrm{s}}\right)$ between points 32 and 45, as well as for points 21, 22 and 23. The violation of constraints in these positions conform to conventional wisdom in two-luminaire design; i.e., these points are not suited for positioning the secondary luminaire.

It is evident that the evaluated populations in subsequent generations were diverse, which gave confidence in the solution found by the GA. The best positions of luminaires $\left(P_{\mathrm{s}}=17, P_{\mathrm{p}}=14\right)$ were also shared by some inferior solutions, demonstrated by the presence of markers of different size and color in Figure 9. To explore further, individual simulations of the design solutions, sharing the same luminaire positions of the best individual in the optimization run, were carried out. The results are summarized in Table 6. Various combinations of luminaire types, $T_{\mathrm{p}}$ and $T_{\mathrm{s}}$ were present in the six further solutions studied. The best design $\left[T_{\mathrm{p}}=\mathrm{B} ; T_{\mathrm{s}}=\mathrm{N}\right]$ was feasible and had the maximum $U_{\mathrm{h}}$; i.e., the minimum $\phi(\mathbf{x})$. Half of the remaining solutions were infeasible. The combination $\left[T_{\mathrm{p}}=\mathrm{B} ; T_{\mathrm{s}}=\mathrm{M}\right]$ violated the constraint for $E_{\mathrm{d}, \text { mean }}$. The combinations $\left[T_{\mathrm{p}}=\mathrm{C} ; T_{\mathrm{s}}=\mathrm{M}\right]$ and $\left[T_{\mathrm{p}}=\mathrm{C} ; T_{\mathrm{s}}=\mathrm{N}\right]$ violated the constraint for $E_{\mathrm{h}, \max }$ but satisfied the other three. 


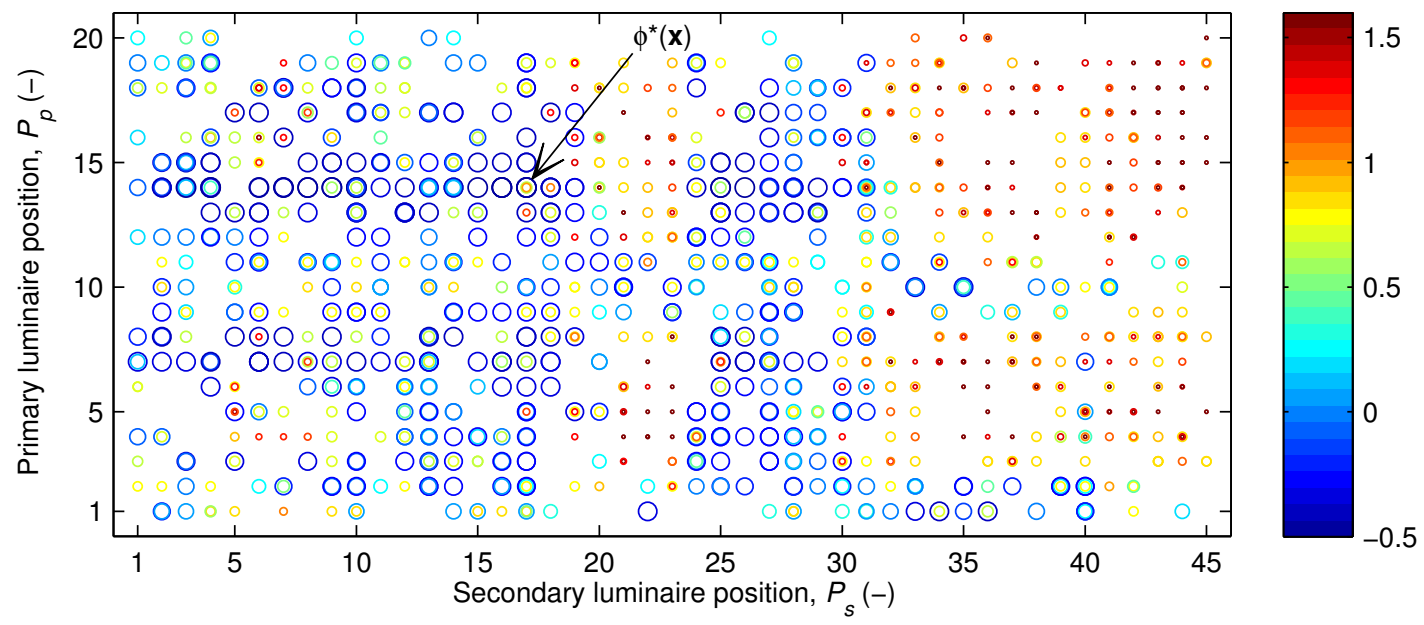

Figure 9: $\phi$-array of fitness in the phenotype space and the location of the best fitness, $\phi^{*}(\mathbf{x})$.

Table 6: Values of fitness and constraint functions of the design solutions sharing the luminaire positions of the best individual in the optimization run.

\begin{tabular}{|c|c|c|c|c|c|c|c|}
\hline$T_{\mathrm{p}}$ & $T_{\mathrm{s}}$ & $\phi(\mathbf{x})$ & $U_{\mathrm{h}}$ & $E_{\mathrm{v}, \max }$ & $E_{\mathrm{h}, \text { mean }}$ & $E_{\mathrm{h}, \max }$ & $E_{\mathrm{d}, \text { mean }}$ \\
\hline \multicolumn{4}{|c|}{$(-)$} & \multicolumn{4}{|c|}{ (lx) } \\
\hline A & $\mathrm{N}$ & -0.5 & 0.5 & 393.67 & 304.68 & 469.05 & 145.62 \\
\hline A & $\mathrm{O}$ & -0.454 & 0.454 & 527.45 & 352.76 & 547.5 & 198.23 \\
\hline B & $\mathrm{M}$ & 0.955 & 0.295 & 285.44 & 261.16 & 527.27 & 81.11 \\
\hline B & $\mathrm{N}$ & -0.515 & 0.515 & 387.73 & 337.73 & 549.46 & 157.92 \\
\hline B & $\mathrm{O}$ & -0.469 & 0.469 & 522.91 & 385.73 & 560.93 & 205.22 \\
\hline C & M & 0.805 & 0.195 & 655.58 & 474.43 & 1031.02 & 106.30 \\
\hline C & $\mathrm{N}$ & 0.645 & 0.355 & 695.18 & 553.01 & 1057.54 & 176.26 \\
\hline
\end{tabular}

The presence of infeasible solutions at the same luminaire positions of the best solution discounts conventional design approaches where either the luminaire positions or the types are kept fixed while the others are allowed to vary. Again, not all feasible solutions in the same positions were optimum. This illustrates the need for optimized lighting design as one proposed solution may satisfy the design criteria; i.e., constraints but may not necessarily be the optimum. Rendered fish-eye perspectives of design 
solutions sharing the same luminaire positions of the best individual $\left[T_{\mathrm{p}}=\mathrm{B} ; T_{\mathrm{s}}=\mathrm{N}\right]$ are illustrated in Figure 10. Figure 10d shows the best design, characterized by uniform lighting with adequate illumination on key interior surfaces whereas the other designs are visibly lacking in one or more design quality indicators.

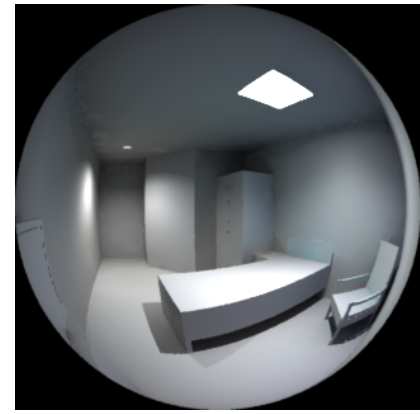

(a) $T_{\mathrm{p}}=\mathrm{A} ; T_{\mathrm{s}}=\mathrm{N}$

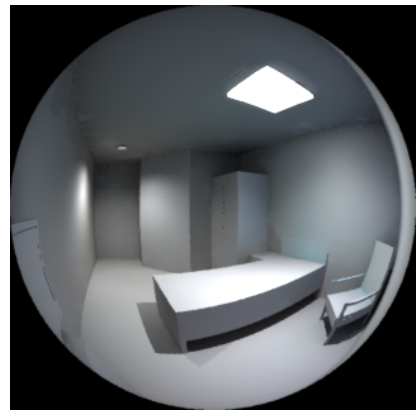

(d) $T_{\mathrm{p}}=\mathrm{B} ; T_{\mathrm{s}}=\mathrm{N}$

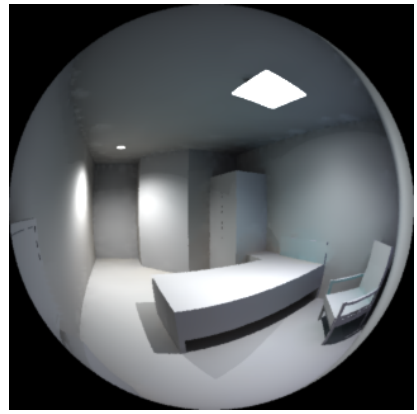

(b) $T_{\mathrm{p}}=\mathrm{A} ; T_{\mathrm{s}}=\mathrm{O}$

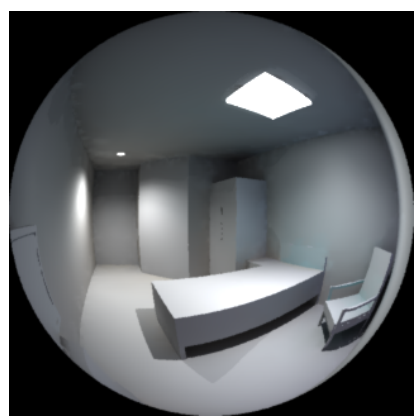

(e) $T_{\mathrm{p}}=\mathrm{B} ; T_{\mathrm{s}}=\mathrm{O}$

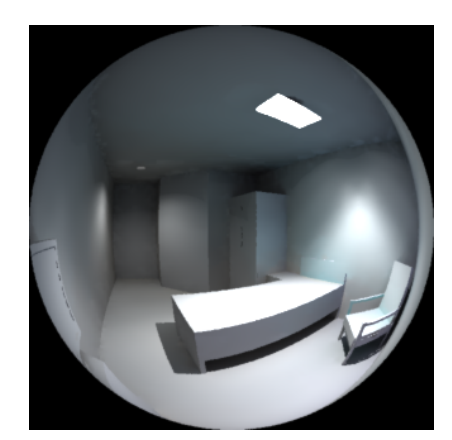

(g) $T_{\mathrm{p}}=\mathrm{C} ; T_{\mathrm{s}}=\mathrm{N}$

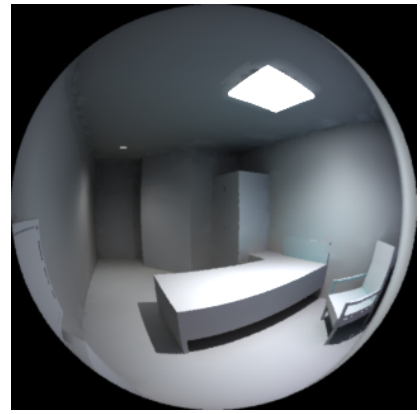

(c) $T_{\mathrm{p}}=\mathrm{B} ; T_{\mathrm{s}}=\mathrm{M}$

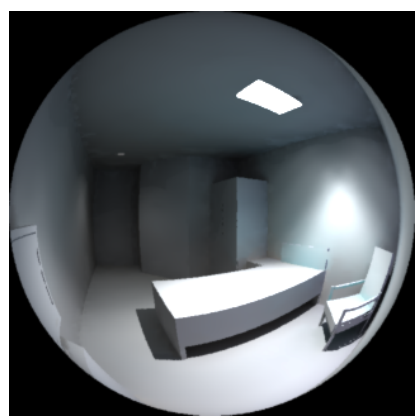

(f) $T_{\mathrm{p}}=\mathrm{C} ; T_{\mathrm{s}}=\mathrm{M}$

Figure 10: Rendered fish-eye perspectives of design solutions sharing the same luminaire positions of the best individual $\left[T_{\mathrm{p}}=\mathrm{B} ; T_{\mathrm{s}}=\mathrm{N}\right]$.

\subsection{Constraint and variable $\phi$-arrays}

The nature of constraint violation and the combinations of luminaire types responsible for the violation are explored further in Figures 11 and 12. $\phi$-array of the violation of the constraint $E_{\mathrm{h} \text {,mean }}$ is shown 
in Figure 11a. There appears to be some similarities between the $\phi$-array and brute-force (Figure 5b) images of $E_{\mathrm{h} \text {,mean }}$. The peaks in the brute-force image can be seen here with lines of dark blue circles $\left(P_{\mathrm{p}}=7\right.$ and 14), in particular at the left half of the image. The presence of orange markers at the top right-hand corner is also consistent with the presence of a number of sharp valleys in the brute-force image. Violations of $E_{\mathrm{h}, \max }$ (Figure 11b), appear to be occurring for some secondary luminaire locations, $P_{\mathrm{s}}$. However, certain regions show a higher tendency of constraint violation such as the regions 41-45 and 19-23, both of which are located next to two major walls. With regard to $E_{\mathrm{d} \text {,mean }}$ in Figure 11c, the trend is clear with $P_{\mathrm{s}}$ regions $20-23$ and 32-45 consistently violating the constraint. This trend can also be seen in the deep valleys of brute-force simulation in Figure $5 \mathrm{~d}$. The peaks in $E_{\mathrm{v}, \text { max }}$ in Figure $5 \mathrm{c}$ can be clearly comprehended in the corresponding $E_{\mathrm{v}, \max } \phi$-array, in which constraints are clearly violated for the region between $P_{\mathrm{s}}$ points 28 and 29. When compared with $\phi$-arrays of luminaire types $T_{\mathrm{p}}$ and $T_{\mathrm{s}}$ in Figure 12, it can be seen that the constraints are violated for all types of luminaires. This discovery suggests that the locations closer to the wall are prone to the violation of $E_{\mathrm{v}, \max }$.
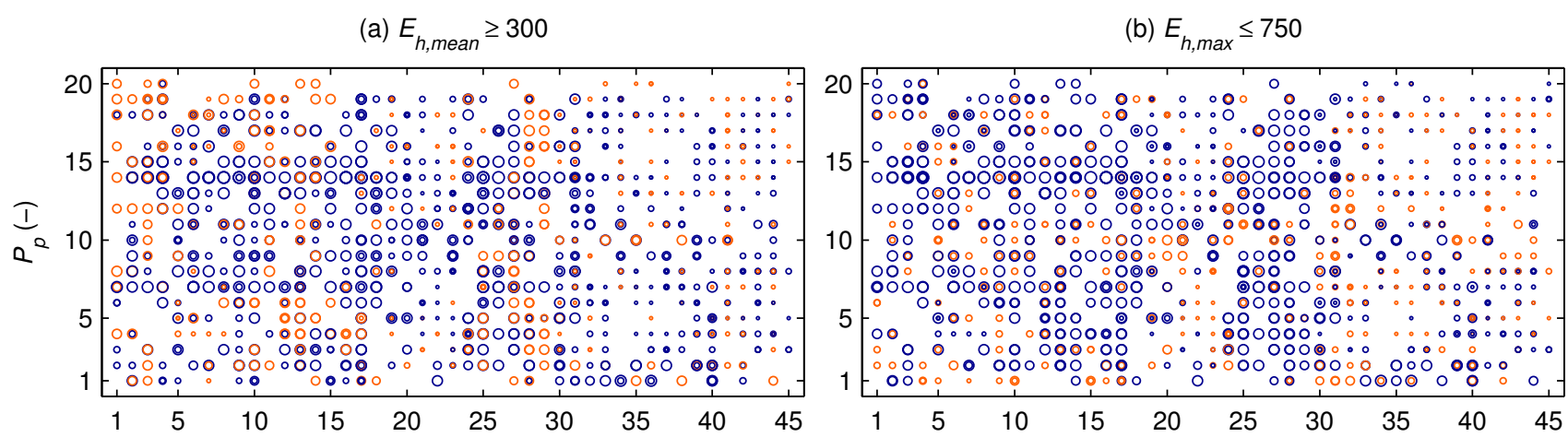

(c) $E_{d, \text { mean }} \geq 100$

(d) $E_{v, \max } \leq 1500$
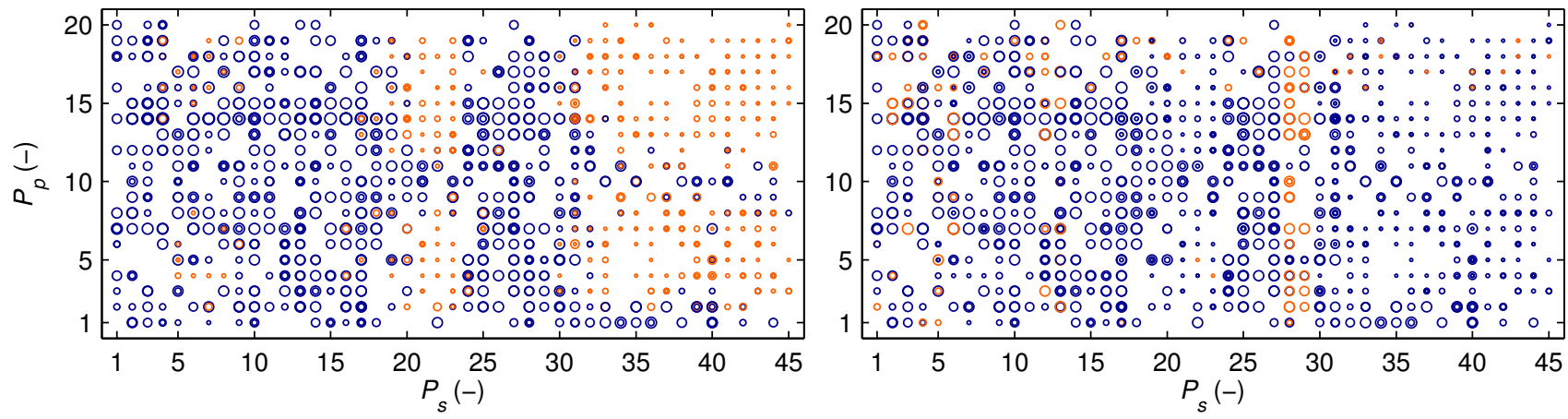

Figure 11: $\phi$-arrays of constraints: (a) mean horizontal illuminance, $E_{\mathrm{h} \text {,mean }}$, (b) maximum horizontal illuminance, $E_{\mathrm{h}, \max }$, (c) mean vertical illuminance on the door, $E_{\mathrm{d} \text {,mean }}$ and (d) maximum vertical illuminance, $E_{\mathrm{v}, \max }$. 
(a) $T_{p}$. Dark blue $=\mathrm{A}$, light blue $=\mathrm{B}$, and orange $=\mathrm{C}$.

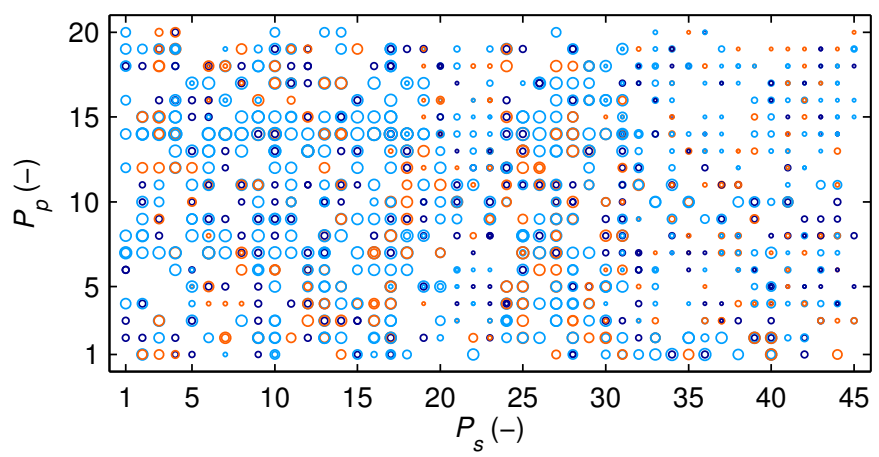

(b) $T_{s}$. Dark blue $=\mathrm{M}$, light blue $=\mathrm{N}$, and orange $=\mathrm{O}$.

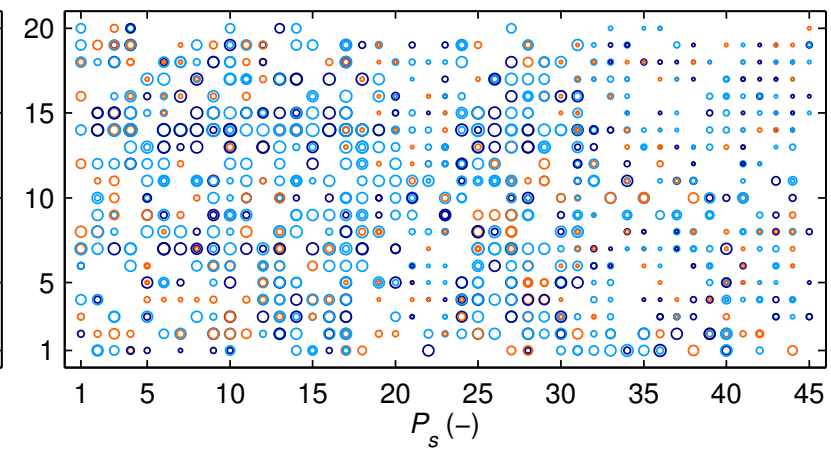

Figure 12: $\phi$-arrays of luminaire types: (a) primary, $T_{\mathrm{p}}$ and (b) secondary, $T_{\mathrm{s}}$.

\subsection{Knowledge discovery}

It is evident from discussions on $\phi$-arrays that such visualizations are quite useful in reinforcing existing knowledge, thereby instilling confidence in decision making through the implied assumption that the model is behaving the way it should. Moreover, new discoveries in the form of improved understanding of the system and its components help to consider the sublime, understated and hidden aspects of optimization. A designer may wish to discard the mathematically optimal solution in favor of an apparently sub-optimal one which may satisfy a different criterion, not considered in the optimization problem. For example, by studying the $\phi$-arrays and Table 6 , a designer may come to a conclusion that the point, $\left[P_{\mathrm{s}}=17 ; P_{\mathrm{p}}=14\right]$, lies in the intersection of prominent lines of feasible designs in the $\phi$-arrays and demonstrates its potential as a region of interest. A close look at Table 6 may reveal to the designer that the difference in $U_{\mathrm{h}}$ (i.e., the goal) between the designs $\left[T_{\mathrm{p}}=\mathrm{B} ; T_{\mathrm{s}}=\mathrm{N}\right]$ and $\left[T_{\mathrm{p}}=\mathrm{B} ; T_{\mathrm{s}}=\mathrm{O}\right]$ is within acceptable limits. Although the design $\left[T_{\mathrm{p}}=\mathrm{B} ; T_{\mathrm{s}}=\mathrm{N}\right]$ is a mathematically better solution because of its lower fitness, the option $\left[T_{\mathrm{p}}=\mathrm{B} ; T_{\mathrm{s}}=\mathrm{O}\right]$ has higher $E_{\mathrm{h} \text {,mean }}$ and $E_{\mathrm{d} \text {,mean }}$. At that point and based on this discovery, the designer may wish to change the goal of the optimization from the maximization of $U_{\mathrm{h}}$ to the maximization of $E_{\mathrm{h} \text {,mean }}$ and $E_{\mathrm{d} \text {,mean }}$, while keeping the other constraints same. [ $T_{\mathrm{p}}=\mathrm{B} ; T_{\mathrm{s}}=\mathrm{O}$ ] can then be the 'preferred' optimal solution, compared to $\left[T_{\mathrm{p}}=\mathrm{B} ; T_{\mathrm{s}}=\mathrm{N}\right]$.

The reduction in the dimensions of the design vector is another great utility of $\phi$-arrays. For example, one can easily conclude from the previous section on constraint violation that secondary luminaire positions in the region 32-45 can be eliminated altogether from similar design problems because of their consistency in violating several constraints. The elimination can result in shorter simulation or computation times; hence saving the designer valuable time in future projects or optimization exercises. Also, this dimension reduction activity can be done on the basis of hierarchical problem solving where small subsets of optimization are done to get a better picture of the problem domain before the final optimization 
is carried out.

\section{Conclusion}

This paper is based on the premise that sub-optimal solutions, typically excluded from the decision making process in evolutionary design optimizations, contain vital information on the underlying behavior of the system. This understanding of system behavior is particularly useful in cases where a small subset of the whole problem is optimized and not all potential design variables are considered due to resource limitations. The visualization of sub-optimal solutions in their corresponding genotype and/or phenotype space can enhance the creative aspects of problem solving by enabling effective design exploration, knowledge discovery and informed decision making.

To enable an effective visualization of sub-optimal solutions, a novel method called $\phi$-array has been developed in this research. $\phi$-arrays are essentially a form of map comprising an arrayed set of data of fitness in its genotype or phenotype space. Information of interest to a designer such as constraint violation or variables are overlaid to form an $n$-dimensional map. The utility of $\phi$-array is tested on an evolutionary design problem in the domain of artificial lighting design of buildings. The test framework comprised a genetic algorithm for effective search of the solution space. Individual designs were evaluated using a computationally expensive physically based raytracing rendering engine called RADIANCE. The design vector consisted of geometric and categorical variables.

Three sets of 4-D $\phi$-arrays were created in this study to visualize fitness, constraints and design variables. Domain knowledge in the form of interrelationships between variables and constraints were extracted which broadly conformed with the trends in partial solution and constraint landscapes of the problem, obtained through brute-force simulations, representing $11.1 \%$ of the total space. Apart from gaining a better understanding of system behavior, non-significant regions were identified in the genotype space suggesting potential for the use of $\phi$-arrays in reducing problem dimensionality in subsequent optimizations.

As a bi-product of this research, some contributions were made in the application of model-based optimization in artificial lighting design, which has conventionally been dominated by the use of simplified methods. The research highlighted the challenging nature lighting design by considering both the psychological and physical aspects of the specialized design of a senior living room. The research also demonstrated that the increasing complexity resulting from our improved understanding of the impact of lighting on occupant health and wellbeing requires the use of advanced search techniques such as optimization, if design performances are to be optimized. With regard to the optimization of artificial lighting designs, the research findings suggested mutation to be more important than crossover as a genetic operation in maintaining the diversity of population and ultimately the success of optimization due 
to the ruggedness of the solution landscape.

Another aspect of optimized design with $\phi$-array visualization is the integration of simulation in design process. In spite of the progress made in simulation (be it energy or physically-based lighting) over past decades, their use in practice remains concentrated among specialists [44]. Instead, simplified methods are predominantly used in most design activities. By demonstrating the usefulness of modelbased optimization in design exploration and knowledge discovery, the community may be swayed in integrating building simulation for performance-based design.

The authors would like to conclude by stating that the potential of $\phi$-arrays in knowledge discovery is only limited by the imagination of the designer. The versatility of $\phi$-arrays in exploring design alternatives means that the method can be applied in many other forms of engineering design, if not all, where creativity is key to producing better performing design solutions.

\section{Future directions and challenges}

With the increasing demand for the consideration of a wide range of variables and goals in design optimization problems, the need for advanced visualization will grow. Enabling a human designer to navigate the solution landscape for domain knowledge extraction during and post optimization will be as important as the efficiency of an algorithm in finding optimum solution(s). The proposed directions of future research include:

- Increasing the dimensions in a $\phi$-array map through the use of advanced information visualization techniques for fine-grained investigations of interrelationships between variable and solution spaces;

- The integration of interactive phenotypic visualizations (e.g. 3D visualization of design variables) with $\phi$-arrays for better contextualization between genotype and phenotype spaces; and

- The investigation of the usability of $\phi$-arrays in industry settings.

Like many other design and visualization methods, the key challenge is the effective integration with design practice. The translation between genotype and phenotype spaces and its contextualization with design goals will require careful considerations in domains where genotype to phenotype translations are not always straightforward.

\section{References}

[1] L. Magnier, F. Haghighat, Multiobjective optimization of building design using TRNSYS simulations, genetic algorithm, and artificial neural network, Building and Environment 45 (3) (2010) 739-746. 
[2] C. Diakaki, E. Grigoroudis, N. Kabelis, D. Kolokotsa, K. Kalaitzakis, G. Stavrakakis, A multi-objective decision model for the improvement of energy efficiency in buildings, Energy 35 (12) (2010) 5483-5496.

[3] H. C. Spindler, L. K. Norford, Naturally ventilated and mixed-mode buildings-Part II: optimal control, Building and Environment 44 (4) (2009) 750-761.

[4] J. Wright, R. Farmani, The simultaneous optimization of building fabric construction, HVAC system size, and the plant control strategy, in: BS2001: Seventh International IBPSA Conference, International Building Performance Simulation Association, Rio de Janeiro, Brazil, 2001, pp. 865-872.

[5] J. Wright, H. Loosemore, The multi-criterion optimization of building thermal design and control, in: BS2001: Seventh International IBPSA Conference, International Building Performance Simulation Association, Rio de Janeiro, Brazil, 2001, pp. 873-880.

[6] L. Zhou, F. Haghighat, Optimization of ventilation system design and operation in office environment, part i: Methodology, Building and Environment 44 (4) (2009) 651-656.

[7] J. C. Miles, G. M. Sisk, C. J. Moore, The conceptual design of commercial buildings using a genetic algorithm, Computers \& Structures 79 (17) (2001) 1583-1592.

[8] P. Geyer, Component-oriented decomposition for multidisciplinary design optimization in building design, Advanced Engineering Informatics 23 (1) (2009) 12-31.

[9] K. W. Park, D. E. Grierson, Pareto-optimal conceptual design of the structural layout buildings using a multicriteria algorithm, Computer-Aided Civil and Infrastructure Engineering 14 (1999) 163-170.

[10] H. Chen, R. Ooka, S. Kato, Study on optimum design method for pleasant outdoor thermal environment using genetic algorithms (GA) and coupled simulation of convection, radiation and conduction, Building and Environment 43 (1) (2008) 18-30.

[11] J. H. Jo, J. S. Gero, Space layout planning using an evolutionary approach, Artificial Intelligence in Engineering 12 (3) (1998) 149-162.

[12] L. G. Caldas, L. K. Norford, A design optimization tool based on a genetic algorithm, Automation in Construction 11 (2) (2002) 173-184.

[13] J. Wright, M. Mourshed, Geometric optimization of fenestration, in: BS2009: Eleventh International IBPSA Conference, International Building Performance Simulation Association, Glasgow, Scotland, 2009, pp. 920-927.

[14] F. Cassol, P. S. Schneider, F. H. França, A. J. S. Neto, Multi-objective optimization as a new approach to illumination design of interior spaces, Building and Environment 46 (2) (2011) 331-338.

[15] J. H. Kämpf, M. Montavon, J. Bunyesc, R. Bolliger, D. Robinson, Optimisation of buildings' solar irradiation availability, Solar Energy 84 (4) (2010) 596-603.

[16] A. Pachamanov, D. Pachamanova, Optimization of the light distribution of luminaries for tunnel and street lighting, Engineering Optimization 40 (1) (2008) 47-65.

[17] E. Elbeltagi, T. Hegazy, D. Grierson, Comparison among five evolutionary-based optimization algorithms, Advanced Engineering Informatics 19 (1) (2005) 43-53.

[18] C. M. Fonseca, P. J. Fleming, An overview of evolutionary algorithms in multiobjective optimization, Evolutionary Computation 3 (1) (2011) 1-16.

[19] W. Wang, H. Rivard, R. Zmeureanu, Floor shape optimization for green building design, Advanced Engineering Informatics 20 (4) (2006) 363-378.

[20] D. E. Grierson, Pareto multi-criteria decision making, Advanced Engineering Informatics 22 (3) (2008) 371-384.

[21] L. Caldas, Generation of energy-efficient architecture solutions applying GENE_ARCH: an evolution-based generative design system, Advanced Engineering Informatics 22 (1) (2008) 59-70.

[22] M. Schena, R. A. Heller, T. P. Theriault, K. Konrad, E. Lachenmeier, R. W. Davis, Microarrays: biotechnology's discovery platform for functional genomics, Trends in Biotechnology 16 (7) (1998) 301-306. 
[23] IESNA, Lighting handbook: Reference \& application, 9th Edition, Illuminating Engineering Society of North America, New York, NY, 2000.

[24] D. Carter, Synthesis of artificial lighting to satisfy multiple design criteria, Building and Environment 18 (3) (1983) $129-134$.

[25] P. Boyce, P. Raynham, The SLL lighting handbook, 1st Edition, Chartered Institution of Building Services Engineers, London, United Kingdom, 2009.

[26] IESNA, IESNA Recommended Practice - Lighting and the Visual Environment for Senior Living, Illuminating Engineering Society of North America, New York, NY, 1998.

[27] G. J. Ward, The RADIANCE lighting simulation and rendering system, in: Proceedings of the 21st annual conference on Computer graphics and interactive techniques, Association for Computing Machinery, 1994, pp. 459-472.

[28] CIBSE, Code for Lighting, Butterworth-Heinemann, Oxford, 2002.

[29] S. Shikder, A. Price, M. Mourshed, Evidence based design of elderly lighting, in: Evidence based design of built healing environment, Loughborough University, Loughborough, United Kingdom, 2009, pp. 87-100.

[30] P. R. Boyce, Human Factors in Lighting, 2nd Edition, Taylor \& Francis, London, United Kingdom, 2003.

[31] CIE, Low vision: Lighting needs for the partially sighted, Tech. Rep. CIE 123-1997, Commission Internationale de l'Eclairage, Vienna, Austria (1997).

[32] CIBSE, Lighting guide 2: Hospitals and health care buildings, Chartered Institution of Building Services Engineers, London, United Kingdom, 2008.

[33] ODPM, The building regulations 2000: Access to and use of buildings: Approved document M: 2004 edition, Office of the deputy prime minister, London, United Kingdom, 2004.

[34] C. A. C. Coello, Theoretical and numerical constraint-handling techniques used with evolutionary algorithms: a survey of the state of the art, Computer Methods in Applied Mechanics and Engineering 191 (11-12) (2002) 1245-1287.

[35] S. Wang, K. Tai, A constraint handling strategy for Bit-Array representation GA in structural topology optimization, Technical report, Massachusetts Institute of Technology, Cambridge, MA (2004).

[36] O. Yeniay, Penalty function methods for constrained optimization with genetic algorithms, Mathematical and Computational Applications 10 (1) (2005) 45-56.

[37] M. M. Mourshed, D. Kelliher, M. Keane, ArDOT: a tool to optimise environmental design of buildings, in: BS2003: Eighth International IBPSA Conference, Vol. 2, Eindhoven, Netherlands, 2003, pp. 919-926.

[38] J. Zhang, X. Yuan, B. P. Buckles, Sample complexity of Real-Coded evolutionary algorithms, in: Proceedings of the sixteenth international Florida artificial intelligence research society conference, Association for the Advancement of Artificial Intelligence (AAAI) Press, St. Augustine, Florida, FL, 2003, pp. 239-243.

[39] R. Haupt, Optimum population size and mutation rate for a simple real genetic algorithm that optimizes array factors, in: IEEE Antennas and Propagation Society International Symposium, Vol. 2, 2000, pp. 1034-1037.

[40] K. D. Jong, An analysis of the behavior of a class of genetic adaptive systems, PhD dissertation, University of Michigan, Ann Arbor, MI (1975).

[41] J. Grefenstette, Optimization of control parameters for genetic algorithms, IEEE Transactions on Systems, Man and Cybernetics 16 (1) (1986) 122-128.

[42] M. Rocha, J. Neves, Preventing premature convergence to local optima in genetic algorithms via random offspring generation, in: Multiple Approaches to Intelligent Systems, Vol. 1611 of Lecture Notes in Computer Science, Springer-Verlag, Berlin, Germany, 2004, pp. 127-136.

[43] J. Potts, T. Giddens, S. Yadav, The development and evaluation of an improved genetic algorithm based on migration and artificial selection, IEEE Transactions on Systems, Man and Cybernetics 24 (1) (1994) 73-86.

[44] C. Reinhart, A. Fitz, Findings from a survey on the current use of daylight simulations in building design, Energy and Buildings 38 (7) (2006) 824-835. 